\title{
Delayed tacrolimus-induced optic neuropathy
}

Muhannad A. Alnabdi, medical student, Yaser M. Al Malik, MBBS, FRCP(c).

\section{ABSTRACT}

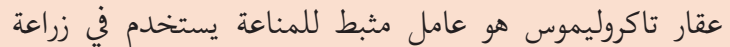

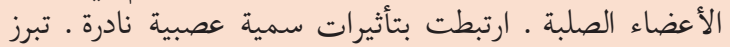

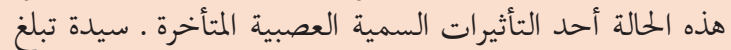

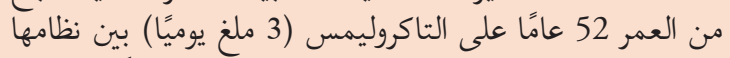

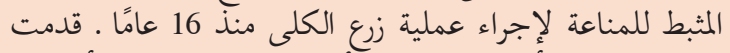

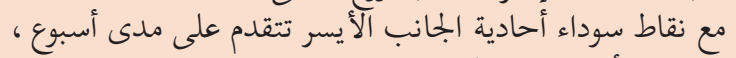

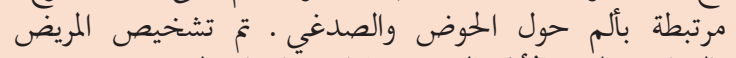

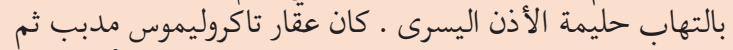

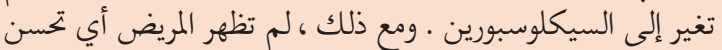

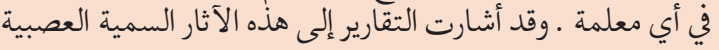

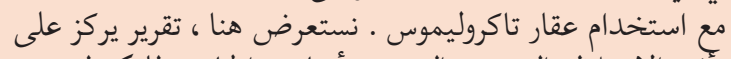

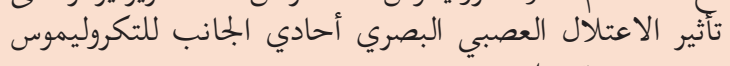
حتى بعد عقد واحد . الاعندا

Tacrolimus is an immunosuppressant agent utilized for solid organ transplantations. It has been associated with rare neurotoxic effects. This case highlights one possible delayed neurotoxic effect. A 52-year-old lady on tacrolimus (3 mg daily) among her immunosuppressive regimen for her kidney transplant 16 year ago. She presented with unilateral left paracentral black dots progressing over a week, associated with periorbital and temporal pain. The patient was diagnosed with left papillitis. Tacrolimus was tapered and then changed to cyclosporine. However, patient did not show any improvement of any parameter. Reports have indicated such neurotoxic effects with Tacrolimus use. Here, the report emphasizes on the unilateral optic neuropathic effect of tacrolimus even after one decade.

Neurosciences 2019; Vol. 24 (4):324-326 doi: 10.17712/nsj.2019.4.20190022

From the College of Medicine (Alnahdi, Al Malik), King Saud bin Abdulaziz University for Health Sciences, and from the Division of Neurology (Al Malik), King Abdulaziz Medical City, National Guard Health Affairs, Riyadh, Kingdom of Saudi Arabia.

Received 1st April 2019 Accepted 27th August 2019.

Address correspondence and reprint request to: Dr. Muhannad A Alnahdi, College of Medicine, King Saud bin Abdulaziz University for Health Sciences, Riyadh, Kingdom of Saudi Arabia. E-mail:m1alnahdi@gmail.com

ORICID ID: https://orcid.org/0000-0003-0839-4131
$\mathrm{T}$ acrolimus, an immunosuppressive agent used in organ transplant, suppresses T-cell development and several cytokines. Several studies have reported its use in preventing allogenic organ transplant rejection, ${ }^{1}$ while others have stated its neurotoxic effects on vision. ${ }^{2-7}$ This case report presents a possible association of unilateral left optic neuropathy with tacrolimus neurotoxicity.

Case Report. Patient information. A 52-year-old female underwent renal transplant due to hypertensive renal failure in 2002. The patient had a history of diabetes (post-transplant), hypertension, dyslipidemia, and osteoarthritis. The immunosuppressive regimen comprised of tacrolimus (FK506, $3 \mathrm{mg}$ daily), cellcept (mycophenolate mofetil, $500 \mathrm{mg}$ twice daily), and prednisolone (5 mg daily). Regular follow-ups were carried out with an ophthalmologist with normal fundal examination and no other complaints. Other medication history included anti-diabetic drugs, antihypertensive drugs, atorvastatin, multivitamins, and esomeprazole, and allergy to lisinopril and azithromycin.

Clinical findings. The patient presented with paracentral black dots in the left eye progressing over a week that plateaued later, and motion aggravated periorbital and temporal pain. The ophthalmologist confirmed left papillitis. Additionally, the patient had left relative afferent pupillary defect (RAPD), early lens cataract, with visual acuity (VA) in right (Rt.) eye $20 \backslash 20$ and left (Lt.) eye 20/60. The patient was referred to neurology for a comprehensive exam and workup. On evaluation, the patient's physical examination showed the same VA with normal extraocular muscles (EOM), pain in the Lt. eye with movement was noted, and Lt. papillitis was still evident. Furthermore, cranial nerves, motor, and cerebellar examinations were unremarkable except for antalgic gait. The patient denied any fever, jaw

Disclosure. The authors declare no conflicting interests, support or funding from any drug company. 


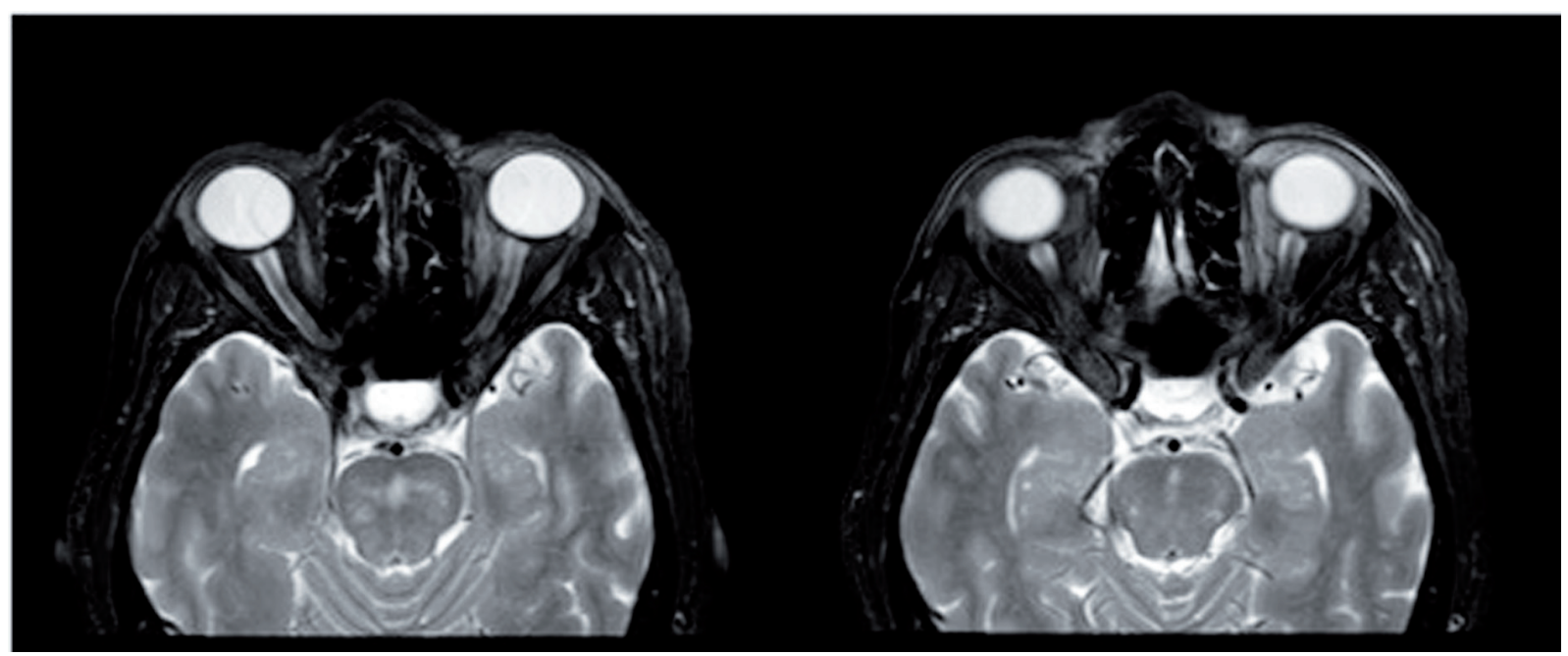

Figure 1 - Magnetic Resonance Imaging images obtained after 4 weeks of presentation. T2 weighted image reflecting the absence of any pathological evidence in the optic pathway that may correlate with neoplastic, inflammatory, or ischemic etiologies.

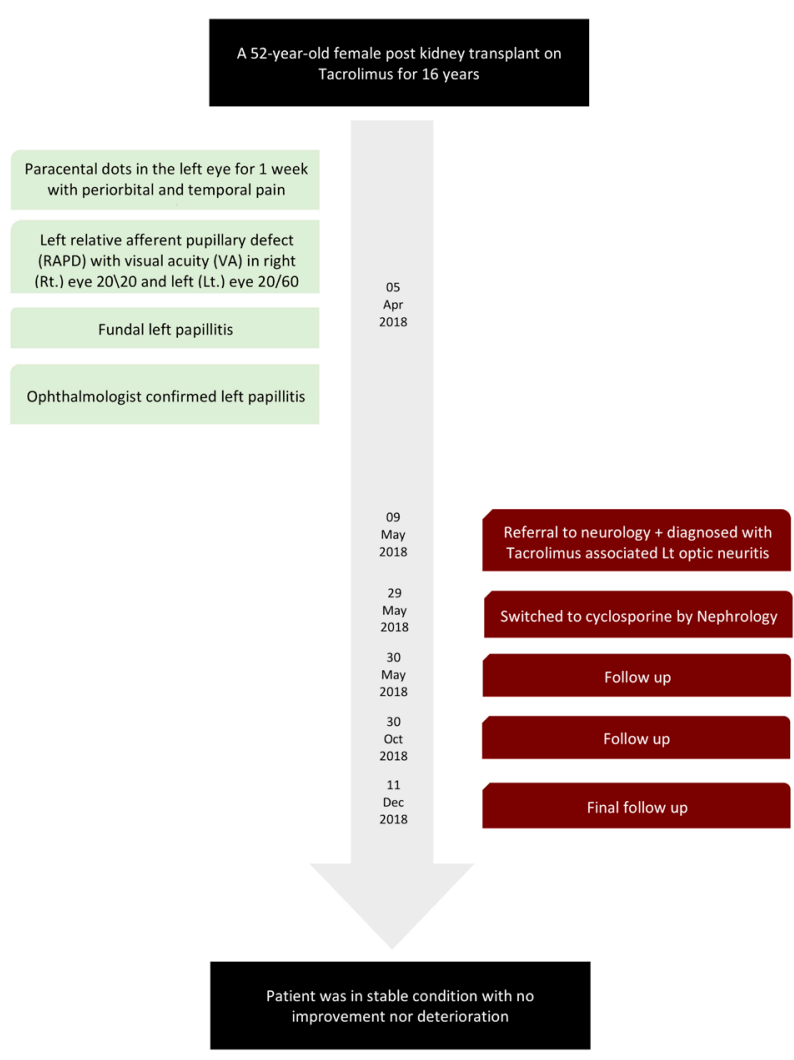

Figure 2 - Timetable demonstrating the sequence of events and case progression. claudication, ptosis, diplopia, recent vaccinations, travel history, weight loss, or any other visual disturbances.

Diagnostic assessment. Extensive investigations were requested. Tacrolimus (FK506) serum level was at $8.9 \mathrm{ng} / \mathrm{mL}$ (target range $5-15 \mathrm{ng} / \mathrm{mL}$ ), and not exceeding $9.4 \mathrm{n} / \mathrm{mL}$ for 5 years. The magnetic resonance imaging (MRI) of orbits and brain was unremarkable for pathological changes, excluding the neoplastic etiologies in the optic pathway, but had multiple white matter foci suggestive of mild chronic ischemic changes (Figure 1). Serology for neuromyelitis optica antibodies, oligo-clonal band antibodies, and cerebrospinal fluid workup tested negative. Laboratory test results showed normal estimated glomerular filtrate rate, C-reactive protein: $9 \mathrm{mg} / \mathrm{L}$ (normal range, $<8 \mathrm{mg} / \mathrm{L}$ ), erythrocyte sedimentation rate: 88 with mild chronic elevation for the last 5 years, otherwise normal. Infectious etiologies were excluded, and stains for cytomegalovirus and mycobacterium were negative.

Therapeutic assessment. The patient was first discharged with a tapered dose of tacrolimus of 1.5 mg daily. Then at 3-week follow-up, tacrolimus was switched to cyclosporine by renal transplant team (Figure 2).

Follow-up and outcomes. Three-week follow-up revealed Lt. RAPD, VA Rt. eye $20 \backslash 20$ and Lt. eye $20 \backslash 120$, visual field (VF) Lt. cecocentral scotoma, and Lt. fundal swelling with normal EOM. At the 6-month follow-up, the patient showed no improvement and symptoms remained similar to the previous state. 
Discussion. Tacrolimus, a calcineurin inhibitor, has shown neurotoxic properties causing optic neuropathy in cases of bone, liver, and islet pancreatic transplantations, even at non-toxic levels. ${ }^{2-7}$ Cortical blindness has been described as a potential adverse effect of tacrolimus use..$^{8-9}$ In this case, neurotoxic effects of tacrolimus was the most probable differential diagnosis after extensive laboratory and imaging workup dismissed any possible role of neoplastic, inflammatory, infectious, or ischemic etiologies. The other probable diagnosis was giant cell arteritis; however, it was excluded by rheumatology team due to the patient's immunosuppressive regimen. The interval between tacrolimus initiation and symptoms onset varied ranging from months to years from previous reports. ${ }^{2-9}$

In this case, the patient was on tacrolimus for 16 years, and had a normal visual follow-up until incidence of painful unilateral optic neuropathy progressing over a week and then plateaued. Therefore, the interval between tacrolimus initiation and visual changes may indicate delayed toxicity. Even after discontinuing the drug no changes were noted in the patients' symptoms, indicating irreversibility of the toxic effect. Venneti et $\mathrm{al}^{6}$ reported a case that showed histopathologic demyelination process due to tacrolimus at non-toxic serum levels. Tacrolimus neurotoxic effect reportedly presented white matter lesions on MRI; however, imaging of our case did not reveal any such lesions consistent with the clinical presentation. ${ }^{10}$ The pathogenesis of tacrolimus-induced optic neuropathy remains unclear, although few reports postulate that ischemia due to vasoconstriction could be the possible etiology. The patient did not regain her previous vision or show any improvement in the visual fields or VA, even after dose tapering or changing to cyclosporine.

In conclusion, tacrolimus has been linked to causing various neurotoxic effects as evident in the case. Unilateral optic neuropathy has been reported throughout the past decade, but the exact etiology remains unclear. However, it is imperative to address the possible delayed toxic role of tacrolimus after long-term use.

Acknowledgements. We would like to thank Editage (www. Editage.com) for English language editing.

\section{References}

1. Christians U, Jacobsen W, Benet LZ, Lampen A. Mechanisms of clinically relevant drug interactions associated with tacrolimus. Clin Pharmacokinet 2002; 41: 813-851.

2. Kessler L, Lucescu C, Pinget M, Charton MN, Mutschler V, Wolf P, et al. Tacrolimus-associated optic neuropathy after pancreatic islet transplantation using a sirolimus/tacrolimus immunosuppressive regimen. Transplantation 2006; 81: 636-637.

3. Brazis PW, Spivey JR, Bolling JP, Steers JL. A case of bilateral optic neuropathy in a patient on tacrolimus (FK506) therapy after liver transplantation. Am J Ophthalmol 2000; 129: 536-538.

4. Kapoor KG, Mirza SN, Gonzales JA, Gibran SK. Visual loss associated with tacrolimus: case report and review of the literature. Cutan Ocul Toxicol 2010; 29: 137-139.

5. Ascaso FJ, Mateo J, Huerva V, Cristóbal JA. Unilateral tacrolimusassociated optic neuropathy after liver transplantation. Cutan Ocul Toxicol 2012; 31: 167-170.

6. Venneti S, Moss HE, Levin MH, Vagefi MR, Brozena SC, Pruitt AA, et al. Asymmetric bilateral demyelinating optic neuropathy from tacrolimus toxicity. J Neurol Sci 2011; 301: 112-115.

7. Rasool N, Boudreault K, Lessell S, Prasad S, Cestari DM. Tacrolimus optic neuropathy. J Neuroophthalmol 2018; 38: 160-166.

8. Devine SM, Newman NJ, Siegel JL, Joseph GJ, Geis TC, Schneider JA, et al. Tacrolimus (FK506)-induced cerebral blindness following bone marrow transplantation. Bone marrow transplantation 1996; 18: 569-572.

9. Shutter LA, Green JP, Newman NJ, Hooks MA, Gordon RD. Cortical blindness and white matter lesions in a patient receiving FK506 after liver transplantation. Neurology 1993; 43: 2417-2418.

10. Steg RE, Kessinger A, Wszolek ZK. Cortical blindness and seizures in a patient receiving FK506 after bone marrow transplantation. Bone marrow transplantation 1999; 23: 959-562. 